PROCEEDINGS OF THE

AMERICAN MATHEMATICAL SOCIETY

Volume 137, Number 11, November 2009, Pages 3905-3910

S 0002-9939(09)09959-6

Article electronically published on June 9, 2009

\title{
COMPLETE REAL HYPERSURFACES IN COMPACT RANK ONE SYMMETRIC SPACES
}

\author{
TATSUYOSHI HAMADA AND KATSUHIRO SHIOHAMA
}

(Communicated by Jon G. Wolfson)

\begin{abstract}
The local classification of real hypersurfaces in a compact rank one symmetric space has been investigated by many people. Making use of the global behavior of geodesics on CROSS, we prove that a complete real hypersurface in a CROSS is a metric sphere if its shape operator and the curvature transformation with respect to the normal have the same eigenspaces at each point of it and if its principal curvatures are constant. We emphasize that our discussion is independent of the choice of the coefficient fields of projective spaces with constant holomorphic sectional curvature.
\end{abstract}

\section{INTRODUCTION}

A well known classical theorem states that a complete totally umbilic hypersurface in the standard $n$-sphere $\mathbf{S}^{n}(c)$ of constant curvature $c>0$ is a metric sphere $S^{n-1}(p ; r)$ centered at $p \in \mathbf{S}^{n}(c)$ with radius $r>0$. In this article, we discuss a natural extension to complete real hypersurfaces with two constant principal curvatures in compact rank one symmetric spaces (abbreviated as CROSS) with constant holomorphic sectional curvature $c>0$. Throughout this paper, let $\widetilde{M}^{n}(c)$ be one of the projective spaces over $\mathbb{C}(\lambda=1), \mathbb{Q}(\lambda=3)$, or $\mathbb{C} a(\lambda=7$ and $k=2)$ of real dimension $n=(\lambda+1) k, k>1$, with the standard metric. The sectional curvature of $\widetilde{M}^{n}(c)$ satisfies

$$
\frac{c}{4} \leq K_{\widetilde{M}} \leq c .
$$

Here, the maximum (minimum, respectively) of $K_{\widetilde{M}}$ is attained at every holomorphic section $(u, \phi u)$, where $u \in T \widetilde{M}$ is a tangent vector and $\phi$ is a structure tensor of type $(1,1)$ (anti-holomorphic section $(u, v)$, where $v$ is orthogonal to holomorphic sections, respectively).

Let $M$ be a complete Riemannian manifold of dimension $(n-1)$ and let $\iota: M \rightarrow$ $\widetilde{M}^{n}(c)$ be an isometric immersion. Let $N$ be a locally defined unit normal field to $M$ along $\iota$. We denote by $A: T M \rightarrow T M$ the shape operator with respect to $N$ such that

$$
A u=-\nabla_{u} N, \quad u \in T M .
$$

Received by the editors October 15, 2008, and, in revised form, March 7, 2009.

2000 Mathematics Subject Classification. Primary 53C20, 53C40.

The research of the first-named author was partially supported by Grant-in-Aid for Scientific Research (C), No. 18540104. The research of the second-named author was partially supported by Grant-in-Aid for Scientific Research (C), No. 19540107. 
Here $\nabla$ is the Levi-Civita connection along $\iota$ of $\widetilde{M}^{n}(c)$.

The tangent space $T_{\tilde{p}} \widetilde{M}^{n}(c)$ to $\widetilde{M}^{n}(c)$ at each point $\tilde{p} \in \widetilde{M}^{n}(c)$ admits a natural orthogonal decomposition:

$$
T_{\tilde{p}} \widetilde{M}^{n}(c)=\langle\xi\rangle \oplus \mathcal{H}_{\xi} \oplus \mathcal{A} \mathcal{H}_{\xi}, \quad \forall \xi \in T_{\tilde{p}} \widetilde{M}^{n}(c), \xi \neq 0 .
$$

Here, we denote by $\left\langle u_{1}, \ldots, u_{m}\right\rangle$ the span of vectors $u_{1}, \ldots, u_{m}$, and

$$
\mathcal{H}_{\xi}:=\left\{u \in \widetilde{M}_{\tilde{p}} \mid K_{\widetilde{M}}(\xi, u)=c\right\}, \quad \mathcal{A H}_{\xi}:=\left\{v \in \widetilde{M}_{\tilde{p}} \mid K_{\widetilde{M}}(\xi, v)=c / 4\right\} .
$$

If $\phi_{1}, \ldots, \phi_{\lambda}$ are the structure tensors of type $(1,1)$ on $\widetilde{M}(c)$, we then have $\mathcal{H}_{N(p)}=$ $\left\langle\phi_{1} N(p), \ldots, \phi_{\lambda} N(p)\right\rangle$ and

$$
\operatorname{dim} \mathcal{H}_{N(p)}=\lambda, \quad \operatorname{dim} \mathcal{A H}_{N(p)}=(\lambda+1)(k-1) .
$$

If $\lambda=1$, then $\phi_{1}$ is the complex structure. The curvature transformation $u \mapsto$ $R(u, \xi) \xi, u \in T_{\tilde{p}} \widetilde{M}^{n}(c)$, for $\xi$ has two eigenspaces: $\mathcal{H}_{\xi}$ with eigenvalue $c$ and $\mathcal{A H}_{\xi}$ with eigenvalue $c / 4$. The tangent space $T_{p} M$ to $M$ at $p \in M$ admits a unique orthogonal decomposition:

$$
T_{p} M=\mathcal{H}_{N(p)} \oplus \mathcal{A H}_{N(p)} .
$$

Our Theorem characterizes the metric spheres in CROSS.

Main Theorem. Let $\iota: M \rightarrow \widetilde{M}^{n}(c)$ be an isometric immersion of a complete Riemannian $(n-1)$-manifold into a projective space $\widetilde{M}^{n}(c)$ of constant holomorphic sectional curvature $c$. Assume that there exist two constants $\kappa_{1}$ and $\kappa_{2}$ such that

$$
c=4 \kappa_{2}^{2}-4 \kappa_{1} \kappa_{2},
$$

and assume that the shape operator A satisfies:

$$
A u=\kappa_{1} u, \forall u \in \mathcal{H}_{N(p)}, \quad A v=\kappa_{2} v, \forall v \in \mathcal{A H}_{N(p)}, \quad \forall p \in M .
$$

Then, there exist a point $\tilde{p} \in \widetilde{M}(c)$ and a positive number $\rho \in(0, \pi / \sqrt{c})$ such that $\iota(M)=S^{n-1}(\tilde{p} ; \rho)$, and $\iota: M \rightarrow \widetilde{M}^{n}(c)$ is an embedding.

R. Takagi 6] first discussed the local shapes of homogeneous real hypersurfaces in $\mathbb{C} P^{n}(c)$ and classified them. Cecil and Ryan [3] proved that if a real hypersurface in $\mathbb{C} P^{n}(c)$ has at most two distinct principal curvatures at each point, then it is an open subset of a metric sphere. Martinez and Perez 5 showed that if the shape operator of a real hypersurface $M \subset \mathbb{Q} P^{n}(c)$ leaves $\mathcal{H}_{N}$ invariant and if the principal curvatures are all constant, then the number $\nu$ of distinct principal curvatures is 2,3 or 4 and $M$ is an open subset of metric spheres centered at a point $(\nu=2)$, a tube over the totally geodesic submanifolds $\mathbb{Q} P^{m}(c), m<k(\nu=3)$ or $\mathbb{C} P^{m}(c)(\nu=4)$. Berndt [1] noticed that the constancy of the principal curvatures of $M \subset \mathbb{Q} P^{n}(c)$ is not necessary. There are a lot of results on the local shapes of such hypersurfaces, for instance [4] and others. The point is whether a certain topological restriction is imposed if completeness is assumed for $M$. Also, the center and radius of a metric sphere should clearly be stated.

In the study of real hypersurfaces in $\widetilde{M}(c)$, the condition that $\phi_{i} N$ for $i=$ $1, \ldots, \lambda$ be an eigenvector of $A$ has played an essential role. The global behavior of geodesics on $\widetilde{M}(c)$ is understood as those on special Blaschke manifolds (for details see [2]), as stated in $\S 2$. The combination of these facts provides us with a general and global treatment of complete real hypersurfaces with two distinct 
principal curvatures in CROSS. In conclusion, every complete real hypersurface in our Theorem is diffeomorphic to $\mathbf{S}^{n-1}$.

\section{Projective spaces}

There are three kinds of totally geodesic submanifolds in $\widetilde{M}(c)$ with constant (holomorphic or anti-holomorphic) sectional curvatures $c$ and $c / 4$. They are $\mathbf{S}^{\lambda+1}(c)$, a hyperplane at infinity $\mathbb{K} P^{k-1}(c)$, and a real projective space $\mathbb{R} P^{n-\lambda}(c / 4)$ of constant sectional curvature $c / 4$.

The injectivity radius $\operatorname{Inj}(\widetilde{M}(c))$ and the diameter $\operatorname{diam}(\widetilde{M}(c))$ of $\widetilde{M}(c)$ satisfy

$$
\operatorname{Inj}(\widetilde{M}(c))=\operatorname{diam}(\widetilde{M}(c))=\frac{\pi}{\sqrt{c}} .
$$

The cut locus $\operatorname{Cut}(\tilde{p})$ to every point $\tilde{p} \in \widetilde{M}(c)$ coincides with the first conjugate locus to $\tilde{p}$. Namely, every geodesic emanating from $\tilde{p}$ of length $\pi / \sqrt{c}$ is minimizing and its endpoint is conjugate to $\tilde{p}$ along it with multiplicity $\lambda$. Moreover, $\operatorname{Cut}(\tilde{p})$ is a totally geodesic submanifold isometric to $\mathbb{K} P^{k-1}(c)=\widetilde{M}^{(\lambda+1)(k-1)}(c)$ and is called the hyperplane at infinity with respect to $\tilde{p}$. Every geodesic on $\widetilde{M}(c)$ is simply closed of length $2 \pi / \sqrt{c}$, along which every antipodal pair is a conjugate pair with multiplicity $\lambda$.

Let $\Gamma(\tilde{p}, \tilde{q})$ for every point $\tilde{q} \in \operatorname{Cut}(\tilde{p})$ be the set of all minimizing geodesics joining $\tilde{p}$ to $\tilde{q}$. Then, $\Gamma(\tilde{p}, \tilde{q})$ forms a $\mathbb{K}$-line $(\mathbb{K}=\mathbb{C}, \mathbb{Q}$ or $\mathbb{C} a)$ which is a totally geodesic submanifold isometric to $\mathbf{S}^{\lambda+1}(c)$. Let $\xi \in T_{\tilde{p}} \widetilde{M}(c)$ be a unit vector and let $\gamma:\left[0,2 \pi / \sqrt{c} \rightarrow \widetilde{M}(c)\right.$ be a simply closed geodesic with $\gamma(0)=\tilde{p}, \gamma^{\prime}(0)=\xi$. Then, $\gamma(t+\pi / \sqrt{c}) \in \operatorname{Cut}(\gamma(t))$ for each $t$, and hence a pair $\left(\gamma(t), \gamma^{\prime}(t)\right)$ determines a unique $\mathbb{K}$-line $\Gamma(\tilde{p}, \gamma(\pi / \sqrt{c}))=\Gamma(\gamma(t), \gamma(t+\pi / \sqrt{c}))$ and its tangent space at $\gamma(t)$ is spanned by $\left\langle\gamma^{\prime}(t)\right\rangle \oplus \mathcal{H}_{\gamma^{\prime}(t)}$. We may express this $\mathbb{K}$-line as $\mathbf{S}_{\gamma^{\prime}(t)}^{\lambda+1}(c)$.

The eigenspace $\mathcal{A H}_{\xi}$ of the curvature transformation for $\xi$ together with $\langle\xi\rangle$ forms the tangent space to the real projective $(n-\lambda)$-space $\mathbb{R} P^{n-\lambda}(c / 4)$. This is a totally geodesic submanifold of $\widetilde{M}(c)$ obtained as $\exp _{\tilde{p}}\langle\xi\rangle \oplus \mathcal{A} \mathcal{H}_{\xi}$. Here, $\mathcal{A H}_{\xi}$ is the orthogonal complement of $\langle\xi\rangle \oplus \mathcal{H}_{\xi}$ in $T_{\widetilde{p}} \widetilde{M}(c)$.

\section{Proof of Theorem}

Under the assumptions in the Theorem, we take an arbitrary fixed point $p \in M$ and $\gamma:[0,2 \pi / \sqrt{c}] \rightarrow \widetilde{M}(c)$ the unit speed simple closed geodesic with $\gamma(0)=$ $\iota(p), \gamma^{\prime}(0)=N(p)$. There is a unique $\mathbb{K}$-line $\mathbf{S}_{N(p)}^{\lambda+1}(c)$ containing $\gamma[0,2 \pi / \sqrt{c}]$. If $u \in \mathcal{H}_{N(p)} \subset T_{\iota(p)} \mathbf{S}_{N(p)}^{\lambda+1}(c)$, then the parallel field $U$ along $\gamma$ generated by $u=$ $U(0)$ satisfies $U(0)=U(2 \pi / \sqrt{c})$ and $U(t) \in \mathcal{H}_{\gamma^{\prime}(t)}$. The parallel field $V$ along $\gamma$ generated by $V(0)=v \in \mathcal{A H}_{N(p)}$ satisfies $V(0)=-V(2 \pi / \sqrt{c})=V(4 \pi / \sqrt{c})$ and $V(t) \in \mathcal{A H}_{\gamma^{\prime}(t)}$.

3.1. Flow curves along $\phi N$. Let $\phi$ be a structure tensor of type $(1,1)$. Let $c:(-\varepsilon, \varepsilon) \rightarrow M$ for a small $\varepsilon>0$ be the flow curve along $\phi N$ such that $c(0):=\iota(p)$, $\dot{c}(s):=\phi N \circ c(s)$. Let $\gamma_{s}:[-\pi / \sqrt{c}, \pi / \sqrt{c}] \rightarrow \widetilde{M}(c)$ for $s \in(-\varepsilon, \varepsilon)$ be a geodesic with $\gamma_{s}(0):=c(s), \gamma^{\prime}(0):=N \circ c(s), \gamma_{0}:=\gamma$. The 1-parameter family of geodesics defines a geodesic variation $\gamma(s, t):=\gamma_{s}(t)$ for $(s, t) \in(-\varepsilon, \varepsilon) \times[\pi / \sqrt{c}, \pi / \sqrt{c}$. The 
variation vector field along each geodesic $\gamma_{s}$ is an $N$-Jacobi field $J_{s}$ whose initial conditions are given as

$$
J_{s}(0)=\phi N \circ c(s), \quad \nabla_{\frac{\partial}{\partial t}} J_{s}(0)=-A \phi N \circ c(s)=-\kappa_{1} \phi N \circ c(s) .
$$

If $E_{s}$ is a unit parallel field along $\gamma_{s}$ with $E_{s}(0)=\phi N \circ c(s)$, then

$$
J_{s}(t)=\left(\cos \sqrt{c} t-\frac{\kappa_{1}}{\sqrt{c}} \sin \sqrt{c} t\right) E_{s}(t)=\sqrt{1+\kappa_{1}^{2} / c} \cdot \cos \sqrt{c}\left(t+\theta_{1}\right) E_{s}(t),
$$

for every $s \in(-\varepsilon, \varepsilon)$. Here, we set

$$
\cos \sqrt{c} \theta_{1}:=\frac{1}{\sqrt{1+\kappa_{1}^{2} / c}}, \quad \sin \sqrt{c} \theta_{1}:=\frac{\kappa_{1} / \sqrt{c}}{\sqrt{1+\kappa_{1}^{2} / c}} .
$$

We define the focal distance and focal point to $N$ along $\gamma$ as follows:

$$
\tilde{p}_{ \pm}:=\exp _{\iota(p)} \rho_{ \pm} N(p), \quad \rho_{+}:=\frac{\pi}{2 \sqrt{c}}-\theta_{1}, \rho_{-}:=-\left(\frac{\pi}{2 \sqrt{c}}+\theta_{1}\right) .
$$

Basic Lemma 3.1. There exists for an arbitrary fixed point $p \in M$ a $\lambda$-dimensional submanifold $\Omega(p) \subset M$ diffeomorphic to $\mathbf{S}^{\lambda}$ such that

(1) $T_{x} \Omega(p)=\mathcal{H}_{N(x)}$, for all $x \in \Omega(p)$;

(2) $\left.\iota\right|_{\Omega(p))}: \Omega(p) \rightarrow \widetilde{M}(c)$ is an embedding;

(3) the image under $\iota$ of $\Omega(p)$ is a small sphere in $\mathbf{S}_{N(p)}^{\lambda+1}(c)$ :

$$
\iota(\Omega(p))=S^{n-1}\left(\tilde{p}_{+} ; \rho_{+}\right) \cap \mathbf{S}_{N(p)}^{\lambda+1}(c)=S^{n-1}\left(\tilde{p}_{-} ; \rho_{-}\right) \cap \mathbf{S}_{N(p)}^{\lambda+1}(c) .
$$

Proof. We define two maps $\varphi^{ \pm}:(-\varepsilon, \varepsilon) \rightarrow \widetilde{M}(c)$ by $\varphi^{ \pm}(s):=\gamma\left(s, \rho_{ \pm}\right), s \in(-\varepsilon, \varepsilon)$. Computation shows that

$$
d \varphi^{ \pm}\left(\frac{\partial}{\partial s}\right)\left(s, \rho_{ \pm}\right)=J_{s}\left(\rho_{ \pm}\right)=0, \quad s \in(-\varepsilon, \varepsilon) .
$$

Therefore, both $\varphi^{+}$and $\varphi^{-}$are constant maps. Setting

$$
\tilde{p}_{ \pm}:=\exp _{\iota(p)} \rho_{ \pm} N(p)=\varphi^{ \pm}(0),
$$

we observe that $d_{\widetilde{M}}\left(\tilde{p}_{+}, \tilde{p}_{-}\right)=\pi / \sqrt{c}$ and that $\gamma_{s}\left(\rho_{ \pm}\right)=\tilde{p}_{ \pm}$for all $s \in(-\varepsilon, \varepsilon)$. The pair $\left(\tilde{p}_{+}, \tilde{p}_{-}\right)$determines a $\mathbb{K}$-line $\Gamma\left(\tilde{p}_{+}, \tilde{p}_{-}\right)=\mathbf{S}_{\gamma^{\prime}(t)}^{\lambda+1}(c), t \in[-\pi / \sqrt{c}, \pi / \sqrt{c}$. Every $\gamma_{s}[-\pi / \sqrt{c}, \pi / \sqrt{c}]$ lies in $\Gamma\left(\tilde{p}_{+}, \tilde{p}_{-}\right)$.

If $\lambda=1$, we then observe from the completeness of $M$ that the flow curve along $\phi_{1} N$ is a small circle of constant curvature $\kappa_{1}$ in $\mathbf{S}_{N(p)}^{2}(c)$. The proof of (2) in this case is later seen in 3.3 .

We may therefore assume that $\lambda>1$. From $\operatorname{Cut}\left(\tilde{p}_{ \pm}\right)=\left\{\tilde{p}_{\mp}\right\}$, we see that $c(-\varepsilon, \varepsilon) \subset \mathbf{S}_{N(p)}^{\lambda+1}(c)$. To construct the desired submanifold, we employ the structure tensors $\phi_{1}, \ldots, \phi_{\lambda}$. The flow curves along the vector fields $\phi_{1} N, \ldots, \phi_{\lambda} N$ define a smooth map from an open set around the origin in $\mathbf{R}^{\lambda}$ into $M$ as follows: Let $c_{i}:(-\varepsilon, \varepsilon) \rightarrow M$ for $i=1, \ldots, \lambda$ be defined inductively by

$$
\begin{aligned}
c_{1}(0) & :=\iota(p), \dot{c}_{1}\left(s_{1}\right):=\phi_{1} N \circ c_{1}\left(s_{1}\right), \\
c_{2}\left(s_{1} ; 0\right) & :=c_{1}\left(s_{1}\right), \dot{c}_{2}\left(s_{1} ; s_{2}\right):=\phi_{2} N \circ c_{2}\left(s_{1} ; s_{2}\right), \\
& \vdots \\
c_{\lambda}\left(s_{1}, \ldots, s_{\lambda-1} ; 0\right) & :=c_{\lambda-1}\left(s_{1}, \ldots ; s_{\lambda-1}\right), \\
\dot{c}_{\lambda}\left(s_{1}, \ldots, s_{\lambda-1} ; s_{\lambda}\right) & :=\phi_{\lambda} N \circ c_{\lambda}\left(s_{1}, \ldots, s_{\lambda-1} ; s_{\lambda}\right) .
\end{aligned}
$$


We then observe that

$$
\left\{\dot{c}_{1}(0), \dot{c}_{2}(0 ; 0), \ldots, \dot{c}_{\lambda}(0, \ldots ; 0)\right\}=\left\{\phi_{1} N(p), \phi_{2} N(p), \ldots, \phi_{\lambda} N(p)\right\}
$$

forms an orthonormal basis for $\mathcal{H}_{N(p)}$ and there exists an open set $\Omega_{0} \subset \mathbf{R}^{\lambda}, 0 \in \Omega_{0}$, such that

$$
\varphi: \Omega_{0} \rightarrow M, \quad \varphi\left(s_{1}, \ldots, s_{\lambda}\right):=c_{\lambda}\left(s_{1}, \ldots ; s_{\lambda}\right)
$$

is an embedding. Setting $\Omega_{0}(p):=\varphi\left(\Omega_{0}\right)$, we see that

$$
\iota\left(\Omega_{0}(p)\right) \subset S^{n-1}\left(\tilde{p}_{+} ; \rho_{+}\right) \cap \mathbf{S}_{N(p)}^{\lambda+1}(c) .
$$

Let $\Omega(p) \subset M$ be the maximal submanifold of $\Omega_{0}(p)$ such that

$$
T_{q} \Omega(p)=\mathcal{H}_{N(q)}, \quad \iota(q) \in S^{n-1}\left(\tilde{p}_{+} ; \rho_{+}\right) \cap \mathbf{S}_{N(p)}^{\lambda+1}(c), \quad \forall q \in \Omega(p) .
$$

It follows from the completeness of $M$ that $N$ is well defined over $\Omega(p)$ and that $\Omega(p)$ is open and closed in $S^{n-1}\left(\tilde{p}_{+} ; \rho_{+}\right) \cap \mathbf{S}_{N(p)}^{\lambda+1}(c)$, and hence

$$
\left.\iota\right|_{\Omega(p)}: \Omega(p) \rightarrow S^{n-1}\left(\tilde{p}_{+} ; \rho_{+}\right) \cap \mathbf{S}_{N(p)}^{\lambda+1}(c)
$$

is surjective. Since the above map is a covering map and its image is simply connected because of $\lambda>1$, it is an embedding.

3.2. Relation between $\kappa_{1}$ and $\kappa_{2}$. Let $a:(-\varepsilon, \varepsilon) \rightarrow M$ be a curve such that $a(0):=\iota(p), \dot{a}(0):=v \in \mathcal{A H}_{N(p)}$ and such that $\dot{a}(s) \in \mathcal{A H}_{N(a(s))}$ for all $s \in(-\varepsilon, \varepsilon)$. We then have a geodesic variation $\delta:(-\varepsilon, \varepsilon) \times[-\pi / \sqrt{c}, \pi / \sqrt{c}] \rightarrow \widetilde{M}$ :

$$
\delta(s, t):=\exp _{a(s)} t N \circ a(s), \quad(s, t) \in(-\varepsilon, \varepsilon) \times[-\pi / \sqrt{c}, \pi / \sqrt{c}] .
$$

Then, the variation vector field $Z$ along $\gamma$ is an $N$-Jacobi field whose initial conditions are given as

$$
Z(0)=v, \quad \nabla_{\frac{\partial}{\partial t}} Z(0)=-A v=-\kappa_{2} v
$$

Setting $V$ as the parallel field along $\gamma$ with $V(0)=v, Z$ is expressed as

$$
Z(t)=\sqrt{1+\frac{4 \kappa_{2}^{2}}{c}} \cdot \cos \frac{\sqrt{c}}{2}\left(t+\theta_{2}\right) V(t)
$$

Here, we set

$$
\cos \frac{\sqrt{c}}{2} \theta_{2}=\frac{1}{\sqrt{1+4 \kappa_{2}^{2} / c}}, \quad \sin \frac{\sqrt{c}}{2} \theta_{2}=\frac{2 \kappa_{2} / \sqrt{c}}{\sqrt{1+4 \kappa_{2}^{2} / c}} .
$$

From (3.1) and (3.4) we observe that (1.3) implies

$$
\cos \sqrt{c} \theta_{1} \cos \sqrt{c} \theta_{2}+\sin \sqrt{c} \theta_{1} \sin \sqrt{c} \theta_{2}=0 .
$$

In particular, we have from (1.3) that

$$
\theta_{1}-\theta_{2}= \pm \frac{\pi}{2 \sqrt{c}}
$$

and hence

$$
\rho_{+}=\frac{\pi}{2 \sqrt{c}}-\theta_{1}=\frac{\pi}{\sqrt{c}}-\theta_{2} .
$$

Therefore, the focal point distance along $\gamma$ has the property

$$
J\left(\rho_{+}\right)=Z\left(\rho_{+}\right)=0, \quad Z\left(\rho_{-}\right) \neq 0 .
$$

Here, $Z\left(\rho_{-}\right)$is tangent to $\operatorname{Cut}\left(\tilde{p}_{+}\right)$at $\tilde{p}_{-}$. 
3.3. Proof of Theorem. We observe from (3.5) that the map $\Phi: M \rightarrow \widetilde{M}$ given by

satisfies that

$$
\Phi(p):=\exp _{\iota(p)} \rho_{+} N(p), \quad \forall p \in M,
$$

$$
d \Phi_{p} u=J_{0}\left(\rho_{+}\right)=0, d \Phi_{p} v=Z\left(\rho_{+}\right)=0, \quad \forall u \in \mathcal{H}_{N(p)}, \forall v \in \mathcal{A H}_{N(p)} .
$$

Therefore $\Phi$ is a constant map. Thus we have

$$
\Phi(M)=\left\{\tilde{p}_{+}\right\}, \quad \iota(M) \subset S^{n-1}\left(\tilde{p}_{+} ; \rho_{+}\right) .
$$

Since $M$ is complete, $\iota(M)$ is open and closed in $S^{n-1}\left(\tilde{p}_{+} ; \rho_{+}\right)$. Further, $\iota: M \rightarrow$ $S^{n-1}\left(\tilde{p}_{+} ; \rho_{+}\right)$is surjective and hence a covering map. Since $S^{n-1}\left(\tilde{p}_{+} ; \rho_{+}\right)$is simply connected, $\iota$ is an embedding. This proves our Theorem.

\section{REFERENCES}

[1] J. Berndt, Real hypersurfaces in quaternionic space forms, J. Reine Angew. Math. 419, 9-26 (1991). MR1116915 (92i:53048)

[2] A. Besse, Manifolds all of whose geodesics are closed, Springer-Verlag, Berlin-New York, 1978. MR 496885 (80c:53044)

[3] T. E. Cecil and P. J. Ryan, Focal sets and real hypersurfaces in complex projective space, Trans. Amer. Math. Soc. 269, 481-499 (1982). MR637703 (83b:53049)

[4] M. Kimura, Real hypersurfaces and complex submanifolds in complex projective space, Trans. Amer. Math. Soc. 296, 137-149 (1986). MR837803 (87k:53133)

[5] A. Martinez and D. Perez, Real hypersurfaces in quaternionic projective space, Ann. Mat. Pura Appl. 145, 355-384 (1986). MR886718 (89a:53062)

[6] R. Takagi, On homogeneous real hypersurfaces in a complex projective space, Osaka J. Math. 10, 495-506 (1973). MR0336660 (49:1433)

Department of Applied Mathematics, Faculty of Sciences, Fukuoka University, 8-19-1 Nanakuma, Fukuoka, 814-0180, Japan - And - Japan Science and Technology Agency, CREST, 5, Sanbancho, Chiyoda-ku, Tokyo, 102-0075, Japan

E-mail address: hamada@holst.sm.fukuoka-u.ac.jp

Department of Applied Mathematics, Faculty of Sciences, Fukuoka University, 8-19-1 Nanakuma, FukUOKa, 841-0180, JaPan 